\title{
FALSE Alarms OF THE CONTACT GLASS-BREAK DETECTORS CAUSED BY THE MULTI-CHAMBER WindoW
}

\author{
Vaclav Mach, Milan Adamek, Irena Drofova, Marta Blahova \& Jan Valouch
}
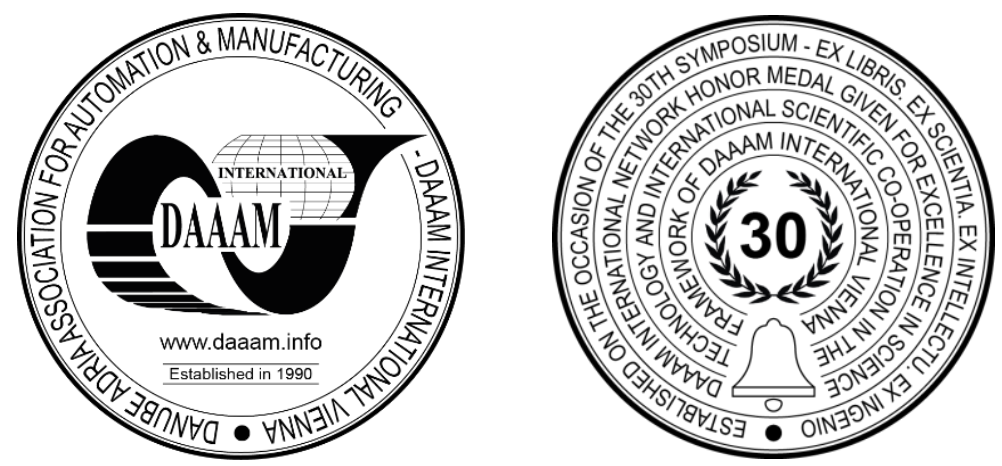

This Publication has to be referred as: Mach, V[aclav]; Adamek, M[ilan]; Drofova, I[rena]; Blahova, M[arta] \& Valouch, J[an] (2020). False Alarms of the Contact Glass-break Detectors Caused by the Multi-chamber Window, Proceedings of the 31st DAAAM International Symposium, pp.0431-0435, B. Katalinic (Ed.), Published by DAAAM International, ISBN 978-3-902734-29-7, ISSN 1726-9679, Vienna, Austria

DOI: $10.2507 / 31$ st.daaam.proceedings.060

\begin{abstract}
The contact glass-break detector is very often marked as very unreliable and these models are not very popular in a security application. The aim of this article is to find out the bottleneck of the glass-break detectors and try to improve their functionality and reliability. The main function is based on the vibration caused by the impact on the glass panel and these vibrations are detected by the detector. However, the biggest issue is the absorption of the vibration by the glass panels and the plastic windows itself. This article should show the vibration transfer difference between the inner and outer glass panels in the multi-chamber window. This experiment should be done according to the current standardization by the two accelerometers on each side of the window. The results should be used for the further improvement of the design and reliability of the contact glass-break detectors on the market.
\end{abstract}

Keywords: Security; Technical Security; Alarm Detector; Glass-break Detector; Multi-chamber Window.

\section{Introduction}

Every person or big company needs to protect its possession, wealth, and money against potential intruders. This protection can be done by the Intruder Alarm System (IAS) and Mechanical Barriers System (MBS). The most convenient variation of protection is the combination of the mentioned systems. The IAS can very easily indicate the intruder and trigger the alarm. [1] Meanwhile, the IBS can very rapidly slow down the progress of the intruder until the security forces arrived at the protected object. Nowadays, the IAS is composed mostly of technical security which is much more effective than physical security. [2]

The technical security of the property can be divided into several layers each representing the level of the intrusion of the intruder. The first and less serious is the perimeter security level which is represented by the area between the protected building and the fence. This area is mostly unprotected, mostly empty, and not interesting for the intruder. The most critical security level between the protected possession and the intruder is the casing protection level which is represented by the walls and openings of the structural building. Everything behind by the walls is called the areal security level where wealth, money, and valuable things can be placed. According to this information, the casing security level is the most important one. 
The casing security level can be divided into three basic parts like walls, doors, and windows. The wall itself cannot be easily destroyed by the intruder without any significant noise and it is practically impossible to break through the wall. The door is very often well protected due to the main entrance and it is the most protected part of the casing security level. The last part is the window which is made from the fragile glass are it is the most vulnerable for the intruder. The windows can be protected by the contact or contactless glass-break detectors which can detect the breaking through it. The current design of the detectors, standardization, and the windows frame is described in detail in the following chapter.

\subsection{Glass-break detectors}

The glass-break detectors are mainly used for the indication of the glass breaking. When the detector is triggered the alarm message is sent to the Control and Indicating Equipment which can send the security forces to the building. [3] As mentioned before, there are two types of glass-break detectors. The first one is focused on the acoustic energy caused by the crushing glass called contactless. The second one is focused on the vibration of the glass panel placed on the protected windows. Both designs are very affected by false alarms which can be triggered by several environmental influences.

Many publications like [4] justifiably evaluating the glass-break detectors as the most unreliable detectors ever due to the high number of false alarms. The other publication [5] tries to point out that the design of the glass-break detector is quite old and it needs innovation.

The publication [6] even tries to improve the signal processing of the contactless glass-break detectors which can reduce up to $20 \%$ of the false alarms. All publication points out the problem of the false alarm of the glass-break detectors. From the practical point of the view, the contact glass-break detector is more convenient with the combination of the mentioned MBS. The contactless glass-break detector of other area detectors can detect the intruder after the breaking trough which can take several minutes. On the other hand, the contact glass-break detector can trigger the alarm at the first impact of the intruder on the window. The combination of the contact glass-break detector and the security window foil is the best combination of the protection for the casing security level. When the intruder hits the window, the alarm is triggered and the intruder must get through the tough foil which takes several minutes according to the foil. According to the mentioned information, this article is focused on the false alarm of the contact glass-break detector and on the elimination of these false alarms.

\section{The current concept of the Glass-break detectors}

Despite the contactless glass-break detector, the contact glass-break detector must be physically placed on the glass panel in the window frame. Every protected window inside of the protected building must have a glass-break detector installed and the device can block a little part of the view from the window. It can be seen by the potential intruder and it can act as prevention. Every contact glass-break detector available on the market must be installed on the inner side of the window where it is protected against the potential intruder and environmental influence. This restriction is very critical for a multi-chamber window where several glass panels are installed into a single-window frame. The presented problem is described in detail in the following chapter.

\section{Energy losses in the window frame}

As mentioned before, the glass-break detector must be installed on the inner side of the window and most of the current types of windows use the multi-chamber technology. This combination is very inappropriate due to the energy which is absorbed by the glass panel and the window frame before it gets to the detector on the other side of the window. The multi-chamber window is done by two independent parts. The first part is composed of two glass panels merged together by the spacer and the glue. The second part is the outer plastic frame with a lot of chambers which makes heat and noise insulation. When the intruder hits the window from the outer side, the energy in form of the vibration must be transferred by the outer glass panel, through the plastic frame to the inner glass panel. The main focus of this article is to try to measure the amount of the absorbed energy produced by the intruder. This absorption should be the main problem that causes false alarms. The experiment for energy absorption is presented and described in the following chapter.

\subsection{Glass-break detectors}

The main experiment should have information about the force differences caused by the intruder between the inner and outer sides of the multi-chamber window. This ration can be used for the calculation of the absorbed energy in percentage. The first question is focused on the device which can be used for the measuring of the small vibration without any negative effect on the inner and outer side of the window. The best device for this type of experiment is the accelerometer which is small, light, cheap, and very accurate. The accelerometer is a device that can measure the acceleration in a given direction. [7] However, the other device must be connected to the accelerometer and it should be able to read and evaluate the information. This device is called the microcontroller, and in this experiment, the ATmega328P embedded inside of the Arduino Uno R3 was chosen. The only feature needed for the microcontroller is the communication interface $\mathrm{I} 2 \mathrm{C}$ which is needed for the communication with the accelerometer. 
The most suitable accelerometer for this experiment is the MPU-6050 for its communication interface and already existing libraries. Moreover, the experiment includes two accelerometers MPU-6050 each for one side of the window. The electronic wiring between the microcontroller and accelerometers for the experiment can be found in the following figure.

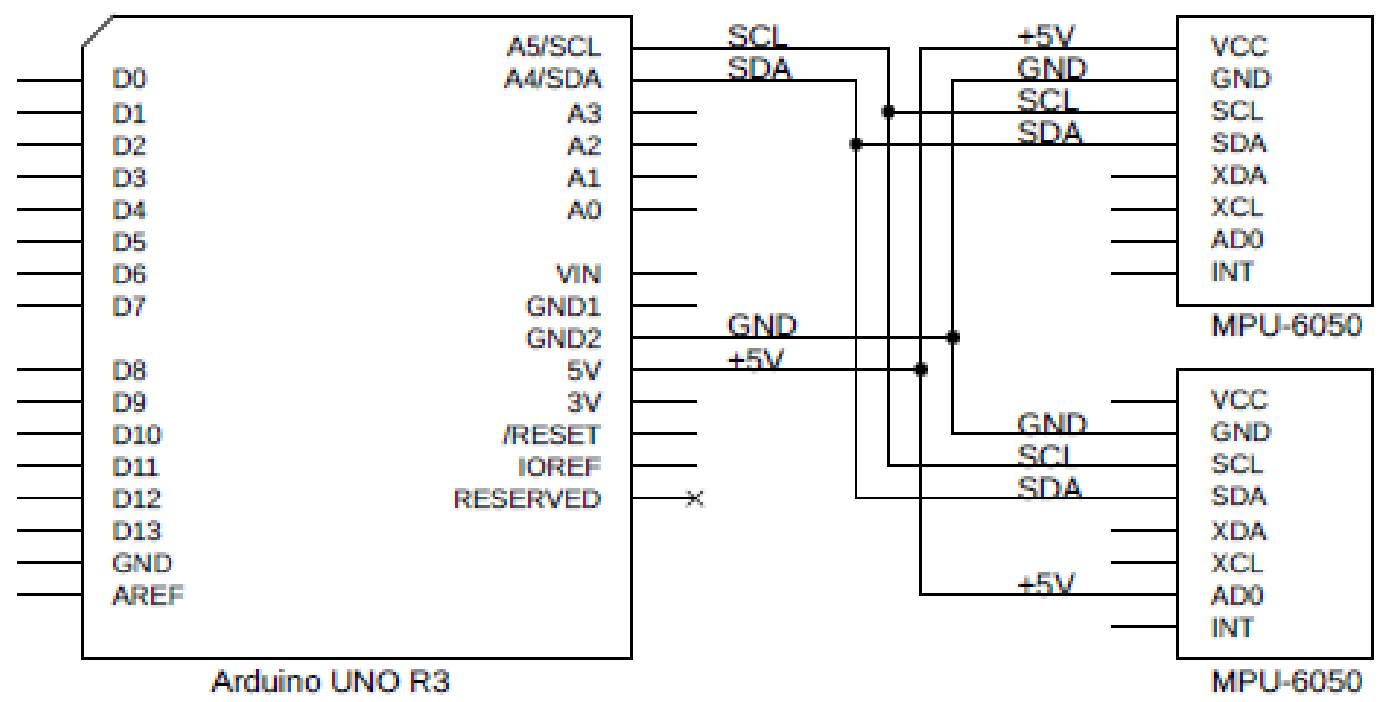

Fig. 1. The schematic connection between the microcontroller and accelerometers

The experiment itself should be done according to the current standardization for the real testing of the contact glassbreak detectors before it gets to the market. This standardization used for this experiment is called CSN CLC/TS 50131 2-7-2: Alarm systems - Intrusion and hold-up systems - Part 2-7-2: Intrusion detectors - Glass break detectors (passive). [8] The standardization has a part which is deals with the testing of the resistance of false alarms which is the main focus of this article. According to the mentioned standardization, the main experiment was constructed.

The experiment is focused on the simulation of the hard objects which can be through on the window without any damage and the detectors should be indicated this event without triggering the alarm. The testing is done on the regular window with the frame and the rubber ball on the pendulum. The rubber ball hits the window at the angle $45^{\circ}$, the length of the pendulum is $1 \mathrm{~m}$, the rubber ball weighs $0,38 \mathrm{~kg}$, and the window is firmly placed in the wall. The experiment is listed in the following figure.

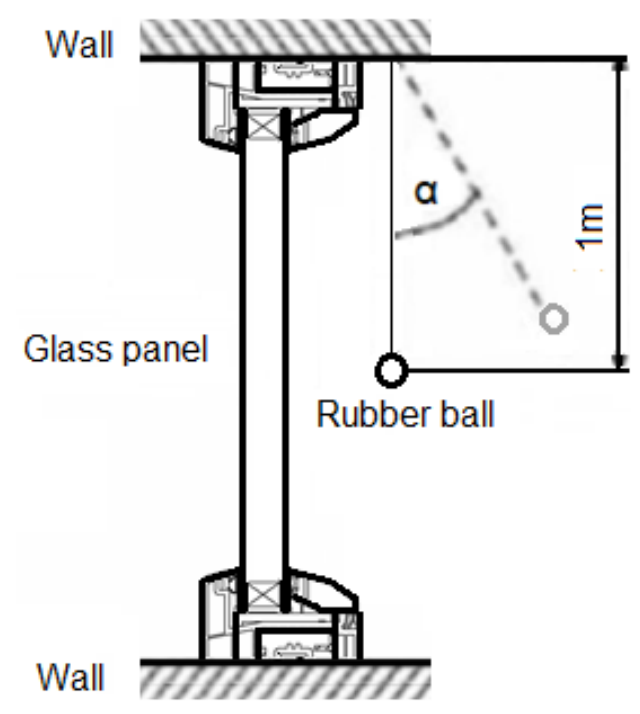

Fig. 2. The design of the experiment

For the sufficient amount of the data, the impact of the rubber ball on the window was done in total 30 times with the standard size of the window $800 \mathrm{~mm}$ x $800 \mathrm{~mm}$, float glass, thickness $6 \mathrm{~mm}$. The maximal value form the outer and inner accelerometer was averaged and the differences were expressed as a percentage. The graphical representation of the energy level of the inner and outer glass panel can be found in the following figure, where blue is the inner and red outer side of the window. 


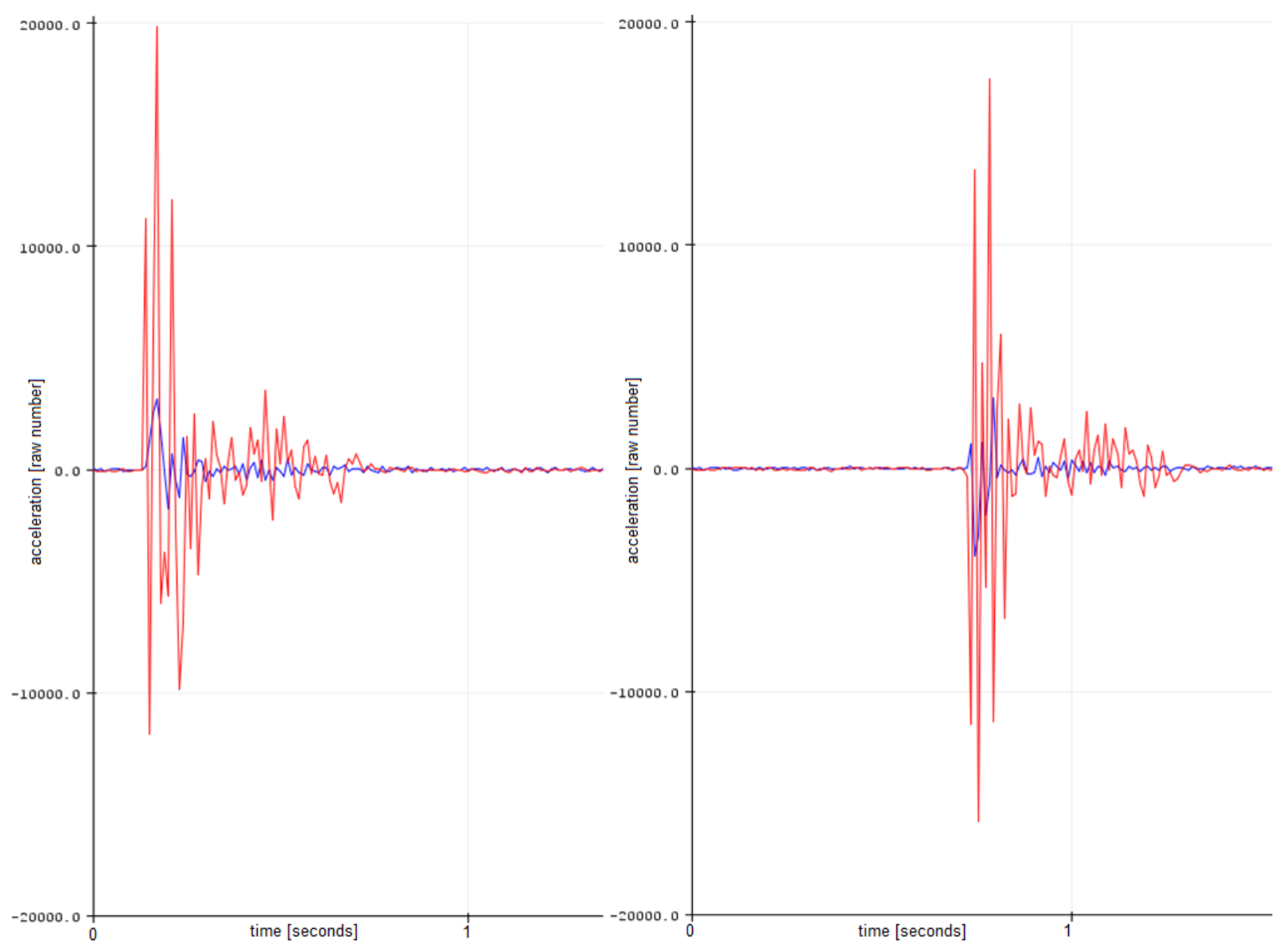

Fig. 3. The energy differences between the inner and outer side of the glass panel

The previous figure shows the differences in the vibration level between the inner and outer sides of the multi-chamber window. Both waveforms were measured and displayed at the same time as can be seen in the figure. This figure is only a demonstration of digital data.

\section{Results and Discussion}

The experiment confirms the huge energy losses inside of the window caused by the multi-chamber construction. The vibrations are absorbed by the soft glue which holds the multi-chamber glass panels together. Moreover, the vibrations are also absorbed by the plastic frame. The amount of energy losses is estimated at $80 \%$. This huge number can very easily cause a huge amount of false alarms and it can lead to the unreliability of the contact glass-break detectors. However, the current construction and the design is cannot be changed, the detector must be installed on the inner side of the window, and the modification of the evaluating program is useless. The only way, how to contact glass-break detectors can be improved is the placement of the physical detector on the outer glass panel which is very difficult to impossible.

\section{Conclusion}

This article is focused on the contact glass-break detectors used in the alarm and the security application. The biggest problem of this topic is the position of the detectors on the inner side of the window. The physical impact on the protected window is done on the outer side of the window and the vibration must travel through the whole window which causes a huge absorption of produced energy. This absorption can lead to an inaccurate evaluation by the alarm detector and it also can lead to a high number of false alarms. The aim of this article was to properly measure the mentioned absorption by the accelerometers according to the current standardization and it proves that the window frame absorbs up to $80 \%$ of the vibration. The main experiment was done using two identical accelerometers each on different sides of the window. The rubber ball was dropped on the window and the energy amount of the created and transferred energy was evaluated by the accelerometers. The experiment showed that the current design of the contact glass-break detectors is very unreliable and inaccurate. The current problem can be solved by the placement of the detector on the inner side of the multi-chamber window. Furthermore, other types of glass panels which are also mentioned in the current standardization can be measured. 


\section{Acknowledgments}

This work was supported by the Ministry of Education, Youth and Sports of the Czech Republic within the National Sustainability Program Project No. LO1303 (MSMT-7778/2014) and also by the European Regional Development Fund under the project CEBIA-Tech ED2.1.00/03.0089 and by the Internal Grant Agency of Tomas Bata University under the project No. IGA/CebiaTech/2020/003.

\section{References}

[1] Landa, J.; Jun, Ch. \& Jun, M. (2017). Implementation of a Remote Real-Time Surveillance Security System for Intruder Detection, 9th International Conference on Measuring Technology and Mechatronics Automation (ICMTMA), doi:10.1109/icmtma.2017.0032

[2] Valouch, J. (2015). The Proposal of Methodology for Evaluating the Effectiveness of Alarm Systems. Applied Mechanics and Materials, pp. 183-88, doi:10.4028/www.scientific.net/amm

[3] Sevčík, J.; Malus, M. \& Svoboda, P. (2014). Large-scale industrial company alarm receiving centre modernization design, WSEAS Transactions on Communications, pp. 587-595, ISSN 1109-2742

[4] Hart, J.; Hartova, V. \& Bradna, J. (2016). Intrusion and Hold -Up Alarm Systems and Their Reliability Glass Break Detection, 6th International Conference on Trends in Agricultural Engineering 7-9 September, pp. 171-174

[5] Gestner, B.; Tanner J. \& Anderson D. (2007). Glass Break Detector Analog Front-End Using Novel Classifier Circuit, International Symposium on Circuits and Systems, pp. 3586-3589, DOI: 10.1109/ISCAS.2007.378528

[6] Nidlova, V. \& Hart, J. (2016). Reliability of detection of Glass Break in Intrusion and Hold-Up Alarm Systems. Advanced Materials and Structural Engineering, pp. 445-448, DOI: 10.1201/b20958-95

[7] Omidalizarandi, M.; Herrmann, R.; Kargoll, B.; Marx, S.; Paffenholz, J. \& Neumann, I. (2020). A validated robust and automatic procedure for vibration analysis of bridge structures using MEMS accelerometers. Journal of Applied Geodesy, 14(3), pp. 327-354, DOI: 10.1515/jag-2020-0010. ISSN 1862-9024

[8] CSN EN 50131-2-7-2 (334591). (2013). Alarm systems - Intrusion and hold-up systems - Part 2-7-2: Intrusion detectors - Glass break detectors (passive), Prague: The Office for Standards, Metrology and Testing 